\title{
Same-Day Breast Cancer Surgery and TARGIT-IORT: Better than Selective Omission of Radiotherapy?
}

\author{
Benjamin D. Smith, $\mathrm{MD}^{1}$, and Henry M. Kuerer, $\mathrm{MD}, \mathrm{PhD}^{2}$ \\ ${ }^{1}$ Department of Radiation Oncology, The University of Texas MD Anderson Cancer Center, Houston, TX; ${ }^{2}$ Breast Surgical \\ Oncology, The University of Texas MD Anderson Cancer Center, Houston, TX
}

Results of the TARGIT-R (retrospective) multi-institutional study on intraoperative radiation therapy (IORT) have been eagerly awaited and are now published in this issue of Annals of Surgical Oncology. ${ }^{1}$ In this case series comprising 667 patients, IORT was delivered using the Intrabeam $^{\text {TM }}$ system (low-kilovoltage X-rays; Carl Zeiss, Oberkochen, Germany) between 2007 and 2013 at 12 North American institutions with active breast cancer programs. The aim of this study was to provide real-world evidence on outcomes of Intrabeam ${ }^{\mathrm{TM}}$ IORT in order to supplement the results of the TARGIT-A randomized clinical trial. ${ }^{2,3}$ Results of the TARGIT-A trial have generated considerable controversy, given small but possibly significant differences in local control between IORT versus whole-breast radiation and concerns that IORT target volumes were too small and IORT radiation doses were too low. ${ }^{4}$ The need for real-world evidence regarding the effectiveness of IORT was underscored by the 2017 update to the American Society for Radiation Oncology (ASTRO) guideline on partial breast irradiation, which recommended that Intrabeam ${ }^{\text {TM }}$ IORT should be used within the context of a prospective registry or clinical trial. ${ }^{5}$ The authors of the TARGIT-R trial are therefore to be commended for heeding this call and for providing this important evidence to help foster a greater understanding regarding the real-world effectiveness of Intrabeam ${ }^{\mathrm{TM}}$ within current practice in the US.

(C) Society of Surgical Oncology 2021

First Received: 9 December 2020

Accepted: 31 December 2020;

Published Online: 15 January 2021

B. D. Smith, MD

e-mail: BSmith3@mdanderson.org
The current report includes 667 patients, and it is notable that it includes only $71 \%$ of the originally reported patient cohort. ${ }^{6}$ Of greatest interest in the current manuscript is the subgroup of 477 patients treated with primary IORT, defined as IORT delivered at the time of segmental mastectomy without additional whole-breast irradiation. In this cohort, median age was 68 years, $94 \%$ of tumors were estrogen receptor-positive, $3 \%$ were lymph node-positive, and only $15 \%$ of tumors were high-grade. Due to these overall favorable characteristics, one would assume that the anticipated risks of in-breast tumor recurrence (IBTR) within 5 years would generally be similar to other clinical trials that enrolled a low-risk patient population. For example, both the PRIME II and CALGB 9343 trials enrolled low-risk patient populations and reported a 5-year IBTR risk of $4 \%$ in patients treated with endocrine therapy alone, compared with $1 \%$ in patients treated with endocrine therapy and whole-breast irradiation. ${ }^{7} 8$ Based on the results of these clinical trials, coupled with the low-risk nature of the population treated with primary IORT on the TARGIT$\mathrm{R}$ study, we expected that the risk of IBTR in the TARGIT$\mathrm{R}$ study would be $<4 \%$ and approach $1 \%$ at 5 years.

However, to our surprise, the 5-year risk of IBTR in the primary IORT cohort of TARGIT-R was $8 \%$ (95\% confidence interval $5.0-10.3 \% ; n=42$ of 477 patients). Even when the authors selected subsets of the primary IORT cohort limited to patients who met criteria established by modern partial breast irradiation guidelines, 5-year IBTR rates ranged from 7.0 to $9.1 \%$, suggesting that more stringent selection criteria would be unlikely to meaningfully reduce the 5-year risk of IBTR following primary IORT reported herein. The authors also noted that noncompliance with endocrine therapy was associated with a higher IBTR risk (hazard ratio 3.67), which is consistent with the conventional wisdom that adjuvant endocrine 
therapy reduces the risk of IBTR. In contrast, among the 116 patients reported in this study who received both IORT and whole-breast radiotherapy (the so-called unintentional boost cohort), only two IBTRs yielded a 5-year risk of $1.7 \%$, similar to the risks we anticipated based on the PRIME II and CALGB 9343 studies.

The very important results of the TARGIT-R prospective registry study suggest that the real-world effectiveness of primary IORT in the US patient population may be inferior to the efficacy of IORT reported in the TARGIT-A trial, in which the 5-year risk of IBTR was $2.1 \%$ (inclusive of both primary IORT and IORT followed by whole-breast irradiation). ${ }^{2}$ The underlying cause of these divergent findings remains unclear, but, in the authors' opinion, the findings of the TARGIT-R registry study should be considered externally valid to a US patient population until proven otherwise.

Moving forward, results from prospective randomized trials now suggest alternative approaches to radiation delivery that can approach the convenience of IORT without sacrificing the proven local control benefits of whole-breast radiation. Specifically, the UK FAST trial showed that once weekly treatment to the whole breast over 5 weeks conferred comparable 10-year local control and toxicity to 5 weeks of daily, Monday through Friday, radiation treatment. Similarly, the UK FAST-Forward trial showed that five daily treatments of radiation to the whole breast conferred comparable 5-year local control and toxicity to 3 weeks of daily, Monday through Friday, radiation treatment. These large, high-quality trials illustrate that the concepts of whole-breast radiation can be extended to very short, convenient fractionation schedules. In our practice, we are now conducting a trial to test the hypothesis that the dose-fractionation of the UK FAST-Forward trial (26 Gy in five daily fractions) can be extended to patients eligible for partial breast irradiation. Given the ongoing uncertainty regarding the real-world effectiveness of Intrabeam $^{\mathrm{TM}}$ IORT, we believe that these short, convenient schedules for external beam radiation represent an attractive current approach to adjuvant treatment.
ACKNOWLEDGMENTS This work was supported by the PH and Fay Etta Robinson Distinguished Professorship in Cancer Research (HMK), the Jay and Lori Eisenberg Distinguished Professorship (BDS), the Cancer Prevention and Research Institute of Texas grant RP160674 (BDS), and the National Cancer Institute Grants R01 CA207216 (BDS) and R01 1CA225646 (BDS), as well as a Cancer Center Support Grant from the National Institutes of Health (NIH; CA16672). Dr. Smith has an equity and royalty interest in Oncora Medical and prior research funding from Varian Medical Systems, unrelated to this topic.

\section{REFERENCES}

1. Valente $\mathrm{S}$, Tendulkar R, Cherian S, et al. TARGIT-R (retrospective): 5-year follow-up on intraoperative radiation therapy (IORT) for breast cancer performed in North America. Ann Surg Oncol. 2021.

2. Vaidya JS, Wenz F, Bulsara M, et al. Risk-adapted targeted intraoperative radiotherapy versus whole-breast radiotherapy for breast cancer: 5-year results for local control and overall survival from the TARGIT — a randomised trial. Lancet. 2014;383:603-13.

3. Vaidya JS, Bulsara M, Baum M, et al. Long term survival and local control outcomes from single dose targeted intraoperative radiotherapy during lumpectomy (TARGIT-IORT) for early breast cancer: TARGIT_a randomised clinical trial. BMJ. 2020;370:m2836.

4. Smith BD, Buchholz TA, Kuerer HM. Intraoperative radiotherapy for early breast cancer. Lancet. 2010;376:1141; author reply $1143-4$.

5. Correa C, Harris EE, Leonardi MC, et al. Accelerated partial breast irradiation: executive summary for the update of an ASTRO evidence-based consensus statement. Pract Radiat Oncol. 2017;7:73-9.

6. Valente SA, Tendulkar RD, Cherian S, et al. TARGIT-R (Retrospective): North American experience with intraoperative radiation using low-kilovoltage X-rays for breast cancer. Ann Surg Oncol. 2016;23:2809-15.

7. Hughes KS, Schnaper LA, Bellon JR, et al. Lumpectomy plus tamoxifen with or without irradiation in women age 70 years or older with early breast cancer: long-term follow-up of CALGB 9343. J Clin Oncol. 2013;31:2382-7.

8. Kunkler IH, Williams LJ, Jack WJ, et al. Breast-conserving surgery with or without irradiation in women aged 65 years or older with early breast cancer (PRIME II): a randomised controlled trial. Lancet Oncol. 2015;16:266-73.

Publisher's Note Springer Nature remains neutral with regard to jurisdictional claims in published maps and institutional affiliations. 